Provided for non-commercial research and education use. Not for reproduction, distribution or commercial use.

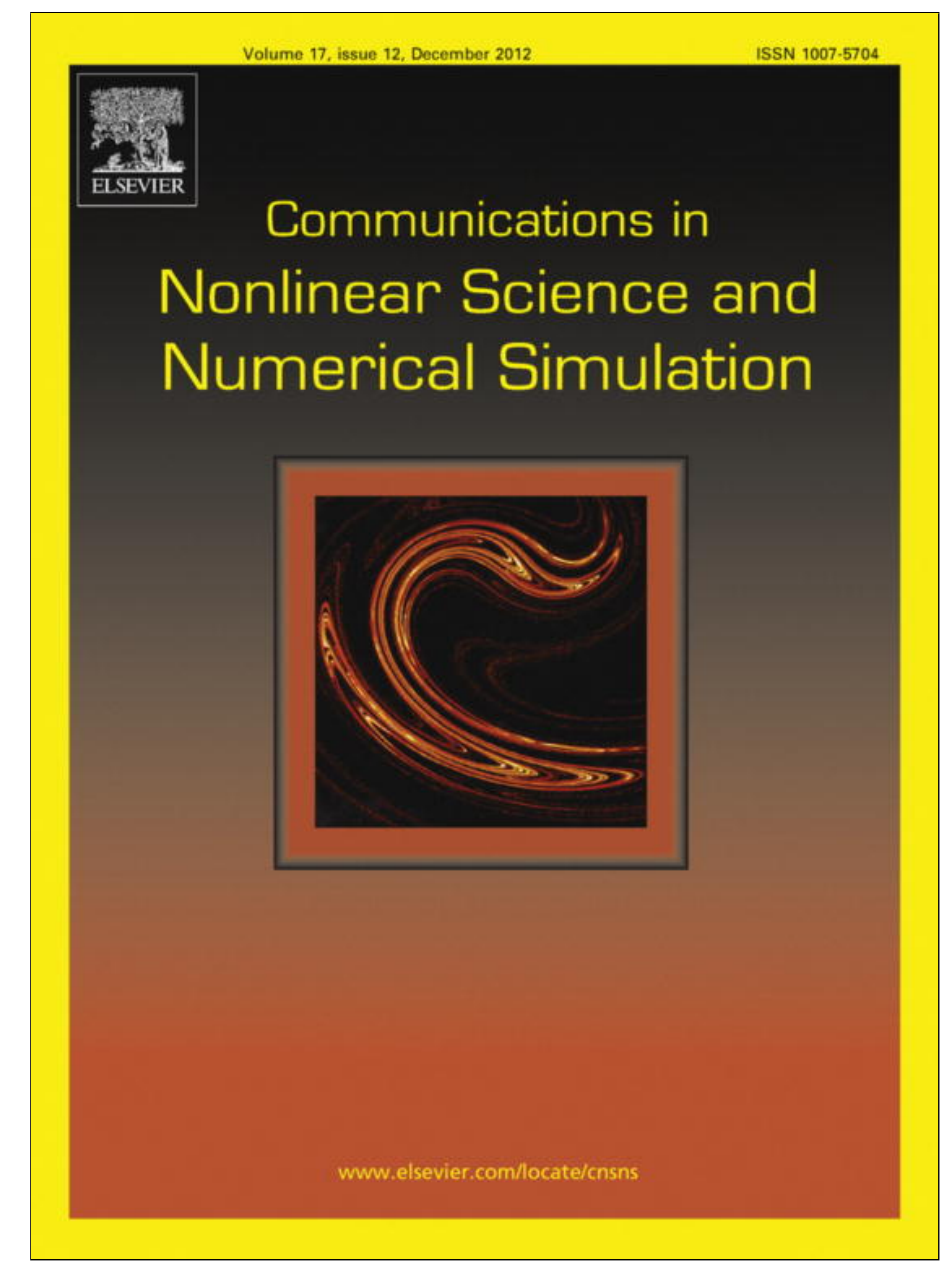

This article appeared in a journal published by Elsevier. The attached copy is furnished to the author for internal non-commercial research and education use, including for instruction at the authors institution and sharing with colleagues.

Other uses, including reproduction and distribution, or selling or licensing copies, or posting to personal, institutional or third party websites are prohibited.

In most cases authors are permitted to post their version of the article (e.g. in Word or Tex form) to their personal website or institutional repository. Authors requiring further information regarding Elsevier's archiving and manuscript policies are encouraged to visit:

http://www.elsevier.com/copyright 


\title{
Non-negative solutions of systems of ODEs with coupled boundary conditions
}

\author{
Gennaro Infante ${ }^{\mathrm{a}, *}$, Feliz M. Minhós ${ }^{\mathrm{b}}$, Paolamaria Pietramala ${ }^{\mathrm{a}}$ \\ a Dipartimento di Matematica, Università della Calabria, 87036 Arcavacata di Rende, Cosenza, Italy \\ ${ }^{\mathrm{b}}$ Department of Mathematics, University of É vora, Rua Romão Ramalho 59, 7000-671 Évora, Portugal
}

\section{A R T I C L E I N F O}

\section{Article history:}

Received 29 January 2012

Received in revised form 29 May 2012

Accepted 31 May 2012

Available online 15 June 2012

\section{Keywords:}

Fixed point index

Cone

System

Non-negative solution

\begin{abstract}
A B S T R A C T
We provide a new existence theory of multiple positive solutions valid for a wide class of systems of boundary value problems that possess a coupling in the boundary conditions. Our conditions are fairly general and cover a large number of situations. The theory is illustrated in details in an example. The approach relies on classical fixed point index.
\end{abstract}

(c) 2012 Elsevier B.V. All rights reserved.

\section{Introduction}

The problem of the existence of positive solutions for systems of local and nonlocal boundary value problems (BVPs) has received an increased attention by researchers, see for example the papers of Agarwal et al. [1-3], Ahmad and Graef [4], Ahmad and Nieto [5], Henderson et al. [14], Lan and Lin [23], Precup [28,29], Yang and Kong [36], Yang and Zhang [37] and references therein. Between systems of BVPs of particular interest are those where the boundary conditions (BCs) are coupled. Systems with coupled BCs can be applied to Lotka-Volterra models, reaction-diffusion phenomena and interaction problems, see for example the works of Amann [7], Leung [24] and Mehmeti and Nicaise [27]. A recent paper in this line of research is the one by Asif and Khan [8], who study the four-point coupled system

$$
\begin{aligned}
& u^{\prime \prime}(t)+f_{1}(t, u(t), v(t))=0, \quad t \in(0,1), \\
& v^{\prime \prime}(t)+f_{2}(t, u(t), v(t))=0, \quad t \in(0,1), \\
& u(0)=0, \quad u(1)=\delta_{12} v\left(\eta_{12}\right), \\
& v(0)=0, \quad v(1)=\delta_{22} u\left(\eta_{22}\right) .
\end{aligned}
$$

The authors prove, via the well-known Guo-Krasnosel'skiı theorem on cone compression-expansion, the existence of one positive solution of the system (1) by means of an associated auxiliary system of Hammerstein integral equations, namely

\footnotetext{
* Corresponding author.

E-mail addresses: g.infante@unical.it (G. Infante), fminhos@uevora.pt (F.M. Minhós), pietramala@unical.it (P. Pietramala).
} 


$$
\begin{aligned}
& u(t)=\int_{0}^{1} F_{1}(t, s) f_{1}(s, u(s), v(s)) d s+\int_{0}^{1} G_{1}(t, s) f_{2}(s, u(s), v(s)) d s \\
& v(t)=\int_{0}^{1} F_{2}(t, s) f_{2}(s, u(s), v(s)) d s+\int_{0}^{1} G_{2}(t, s) f_{1}(s, u(s), v(s)) d s .
\end{aligned}
$$

An integral representation of the type (2) is also used in the papers [9,38]. Yuan et al. in [38] study, by means of a nonlinear alternative of Leray-Schauder type and the Guo-Krasnosel'skiı fixed-point theorem, the existence of one or two positive solutions for a semi-positone system of fractional differential equations subject to four-point BCs. In [9] Cui and Sun study, via fixed point index theory, the existence of one positive solution for the system

$$
\begin{array}{ll}
u^{\prime \prime}(t)+f_{1}(t, u(t), v(t))=0, & t \in(0,1), \\
v^{\prime \prime}(t)+f_{2}(t, u(t), v(t))=0, & t \in(0,1), \\
u(0)=0, & u(1)=\beta_{12}[v],
\end{array}
$$

where $\beta_{i j}[\cdot]$ are linear functionals defined via (positive) Stieltjes measures.

We mention that Stieltjes integrals are also used in the framework of nonlinear coupled BCs in the paper of Kang and Wei [18] (where the Leggett and Williams fixed point theorem is used) and in two recent papers of Goodrich [11,12] (who uses the Guo-Krasnosel'skiı fixed-point theorem); one interesting feature of [11,12] is the possibility to use signed measures, in the line of the paper by Webb and Infante [32].

Here we provide a new approach for a wide class of systems of BVPs that possess, along the coupling in the nonlinearities of the differential equations, also a coupling in the BCs and we prove, under suitable conditions, existence of multiple nonnegative solutions. Our idea is to give an existence theory valid for systems of perturbed Hammerstein integral equations of the type

$$
\begin{aligned}
& u(t)=\gamma_{11}(t) \beta_{11}[u]+\gamma_{12}(t) \beta_{12}[v]+\int_{0}^{1} k_{1}(t, s) g_{1}(s) f_{1}(s, u(s), v(s)) d s, \\
& v(t)=\gamma_{21}(t) \beta_{21}[v]+\gamma_{22}(t) \beta_{22}[u]+\int_{0}^{1} k_{2}(t, s) g_{2}(s) f_{2}(s, u(s), v(s)) d s,
\end{aligned}
$$

where $\gamma_{i j}$ are continuous functions and $\beta_{i j}[\cdot]$ are linear functionals defined via Stieltjes measures. A system of perturbed Hammerstein integral equations similar to (4) is investigated by Infante and Pietramala in [16], with the intent of dealing with BVPs with nonlinear BCs, allowing a coupling in the nonlinearities $f_{1}$ and $f_{2}$ but not in the BCs. The methodology of [16] relies on an extensions of the results of [32] to the context of systems.

We illustrate our theory with an example of a system of second and fourth order ordinary differential equations

$$
\begin{aligned}
& u^{\prime \prime}(t)+g_{1}(t) f_{1}(t, u(t), v(t))=0, \quad t \in(0,1), \\
& v^{(4)}(t)=g_{2}(t) f_{2}(t, u(t), v(t)), \quad t \in(0,1),
\end{aligned}
$$

subject to the nonlocal boundary conditions

$$
\begin{aligned}
& u(0)=\beta_{11}[u], \quad u(1)=\beta_{12}[v], \\
& v(0)=\beta_{21}[v], \quad v(1)=0, \quad v^{\prime \prime}(0)=0, \quad v^{\prime \prime}(1)+\beta_{22}[u]=0 .
\end{aligned}
$$

The system of ordinary differential equations (5), with local BCs, can be used as a model for the stationary states of a onedimensional bridge, with a coupling between the cable and the roadbed. Here the cable is seen as vibrating string and the roadbed as a vibrating beam, see for example the papers of Lazer and McKenna [20], Lü et al. [25], Sun [30] and the doctoral thesis of Matas [26]. The boundary conditions (6) involve functionals of the form

$$
\beta_{i j}[w]=\int_{0}^{1} w(s) d B_{i j}(s)
$$

and include, as special cases, $m$-point and integral conditions, when

$$
\beta_{i j}[w]=\sum_{j=1}^{m} \delta_{i j} w\left(\eta_{i j}\right) \quad \text { and } \quad \beta_{i j}[w]=\int_{0}^{1} \delta_{i j}(s) w(s) d s .
$$

For previous work on Riemann-Stieltjes integral BCs we refer the reader, for example, to the papers of Karakostas and Tsamatos [19] and Webb [31,33]. We point out that nonlocal conditions have a physical interpretation; for example the coupled condition

$$
u(0)=u(1)=v(1)=v^{\prime \prime}(0)=v(0)=0, \quad v^{\prime \prime}(1)+\delta u(\eta)=0,
$$

models a feedback control mechanism, where the bending moment in the right end of the beam is related to the displacement registered in a point $\eta$ of the string. 
We prove our results by means of the classical fixed point index theory (see for example the review of Amann [6] and the book of Guo and Lakshmikantham [13]) and also make use of ideas from the papers [16,17,32].

\section{Positive solutions for systems of integral equations}

In order to utilize the classical fixed point index theory to find positive solutions of the system of integral equations

$$
\begin{aligned}
& u(t)=\gamma_{11}(t) \beta_{11}[u]+\gamma_{12}(t) \beta_{12}[v]+\int_{0}^{1} k_{1}(t, s) g_{1}(s) f_{1}(s, u(s), v(s)) d s, \\
& v(t)=\gamma_{21}(t) \beta_{21}[v]+\gamma_{22}(t) \beta_{22}[u]+\int_{0}^{1} k_{2}(t, s) g_{2}(s) f_{2}(s, u(s), v(s)) d s,
\end{aligned}
$$

we make the following hypotheses on the terms that occur in (7):

- For every $i=1,2, f_{i}:[0,1] \times[0, \infty) \times[0, \infty) \rightarrow[0, \infty)$ satisfies Carathéodory conditions, that is, $f_{i}(\cdot, u, v)$ is measurable for each fixed $(u, v)$ and $f_{i}(t, \cdot, \cdot)$ is continuous for almost every (a.e.) $t \in[0,1]$, and for each $r>0$ there exists $\phi_{i, r} \in L^{\infty}[0,1]$ such that

$f_{i}(t, u, v) \leqslant \phi_{i, r}(t) \quad$ for $u, v \in[0, r]$ and a.e. $t \in[0,1]$.

- For every $i=1,2, k_{i}:[0,1] \times[0,1] \rightarrow[0, \infty)$ is measurable, and for every $\tau \in[0,1]$ we have

$$
\lim _{t \rightarrow \tau}\left|k_{i}(t, s)-k_{i}(\tau, s)\right|=0 \quad \text { for a.e. } s \in[0,1] \text {. }
$$

- For every $i=1,2$, there exist a subinterval $\left[a_{i}, b_{i}\right] \subseteq[0,1]$, a function $\Phi_{i} \in L^{\infty}[0,1]$, and a constant $c_{i} \in(0,1]$, such that

$$
\begin{aligned}
& k_{i}(t, s) \leqslant \Phi_{i}(s) \quad \text { for } t \in[0,1] \text { and a.e. } s \in[0,1], \\
& k_{i}(t, s) \geqslant c_{i} \Phi_{i}(s) \quad \text { for } t \in\left[a_{i}, b_{i}\right] \text { and a.e. } s \in[0,1] .
\end{aligned}
$$

- For every $i=1,2, g_{i} \Phi_{i} \in L^{1}[0,1], g_{i} \geqslant 0$ a.e., and $\int_{a_{i}}^{b_{i}} \Phi_{i}(s) g_{i}(s) d s>0$.

- For every $i, j=1,2, \beta_{i j}[\cdot]$ is a linear functional given by

$$
\beta_{i j}[w]=\int_{0}^{1} w(s) d B_{i j}(s)
$$

involving Riemann-Stieltjes integrals; $B_{i j}$ is of bounded variation and $d B_{i j}$ is a positive measure.

- For every $i, j=1,2, \gamma_{i j} \in C[0,1], \gamma_{i j}(t) \geqslant 0$ for every $t \in[0,1], \beta_{i 1}\left[\gamma_{i 1}\right]<1$ and there exists $c_{i j} \in(0,1]$ such that

$$
\gamma_{i j}(t) \geqslant c_{i j}\left\|\gamma_{i j}\right\|_{\infty} \text { for every } t \in\left[a_{i}, b_{i}\right]
$$

where $\|w\|_{\infty}:=\max \{|w(t)|, t \in[0,1]\}$.

We work in the space $C[0,1] \times C[0,1]$ endowed with the norm

$$
\|(u, v)\|:=\max \left\{\|u\|_{\infty},\|v\|_{\infty}\right\}
$$

Let

$$
\widetilde{K}_{i}:=\left\{w \in C[0,1]: w(t) \geqslant 0 \text { for } t \in[0,1] \text { and } \min _{t \in\left[a_{i}, b_{i}\right]} w(t) \geqslant \tilde{c}_{i}\|w\|_{\infty}\right\}
$$

where $\tilde{c}_{i}=\min \left\{c_{i}, c_{i 1}, c_{i 2}\right\}$, and consider the cone $K$ in $C[0,1] \times C[0,1]$ defined by

$$
K:=\left\{(u, v) \in \widetilde{K}_{1} \times \widetilde{K}_{2}\right\} .
$$

For a positive solution of the system (7) we mean a solution $(u, v) \in K$ of (7) such that $\|(u, v)\|>0$.

Under our assumptions, we show that the integral operator

$$
T(u, v)(t)=\left(\begin{array}{l}
T_{1}(u, v)(t) \\
T_{2}(u, v)(t)
\end{array}\right):=\left(\begin{array}{l}
\gamma_{11}(t) \beta_{11}[u]+\gamma_{12}(t) \beta_{12}[v]+F_{1}(u, v)(t) \\
\gamma_{21}(t) \beta_{21}[v]+\gamma_{22}(t) \beta_{22}[u]+F_{2}(u, v)(t)
\end{array}\right),
$$

where

$$
F_{i}(u, v)(t):=\int_{0}^{1} k_{i}(t, s) g_{i}(s) f_{i}(s, u(s), v(s)) d s,
$$

leaves the cone $K$ invariant and is compact.

Lemma 1. The operator (8) maps $K$ into $K$ and is compact. 
Proof. Take $(u, v) \in K$ such that $\|(u, v)\| \leqslant r$. Then we have, for $t \in[0,1]$,

$$
T_{1}(u, v)(t)=\gamma_{11}(t) \beta_{11}[u]+\gamma_{12}(t) \beta_{12}[v]+\int_{0}^{1} k_{1}(t, s) g_{1}(s) f_{1}(s, u(s), v(s)) d s
$$

therefore

$$
\left\|T_{1}(u, v)\right\| \leqslant\left\|\gamma_{11}\right\|_{\infty} \beta_{11}[u]+\left\|\gamma_{12}\right\|_{\infty} \beta_{12}[v]+\int_{0}^{1} \Phi_{1}(s) g_{1}(s) f_{1}(s, u(s), v(s)) d s .
$$

Then we obtain

$$
\min _{t \in\left[a_{1}, b_{1}\right]} T_{1}(u, v)(t) \geqslant c_{11}\left\|\gamma_{11}\right\|_{\infty} \beta_{11}[u]+c_{12}\left\|\gamma_{12}\right\|_{\infty} \beta_{12}[v]+c_{1} \int_{0}^{1} \Phi_{1}(s) g_{1}(s) f_{1}(s, u(s), v(s)) d s \geqslant \tilde{c_{1}}\left\|T_{1}(u, v)\right\| .
$$

Hence we have $T_{1}(u, v) \in \widetilde{K}_{1}$. In a similar manner we proceed for $T_{2}(u, v)$.

Moreover, the map $T$ is compact since the components $T_{i}$ are sum of two compact maps: the compactness of $F_{i}$ is wellknown and, since $\gamma_{i 1}$ and $\gamma_{i 2}$ are continuous, the perturbation $\gamma_{i 1}(t) \beta_{i 1}[u]+\gamma_{i 2}(t) \beta_{i 2}[v]$ maps bounded sets into bounded subsets of a finite dimensional space.

We use the following (relative) open bounded sets in $K$ :

$$
K_{\rho}=\{(u, v) \in K:\|(u, v)\|<\rho\},
$$

and

$$
V_{\rho}=\left\{(u, v) \in K: \min _{t \in\left[a_{1}, b_{1}\right]} u(t)<\rho \text { and } \min _{t \in\left[a_{2}, b_{2}\right]} v(t)<\rho\right\}
$$

The set $V_{\rho}$ (in the context of systems) was introduced by Infante and Pietramala [15] and is equal to the set called $\Omega^{\rho / c}$ by Franco, Infante and O'Regan [10]. $\Omega^{\rho / c}$ is an extension to the case of systems of a set given by Lan [22]. The advantage of the notation $V_{\rho}$ is that sheds light on the fact (see also the paper by Infante and Webb [17]) that choosing $c$ as large as possible provides a weaker condition to be satisfied by the functions $f_{i}$ in Lemmas 3 and 4 . Note that $K_{\rho} \subset V_{\rho} \subset K_{\rho / c}$, where $c=\min \left\{\tilde{c_{1}}, \tilde{c_{2}}\right\}$. We denote by $\partial K_{\rho}$ and $\partial V_{\rho}$ the boundary of $K_{\rho}$ and $V_{\rho}$ relative to $K$.

In the next Lemma we make use of the notation

$$
\mathscr{K}_{i j}(s):=\int_{0}^{1} k_{i}(t, s) d B_{i j}(t), \quad i, j=1,2,
$$

and we prove that the index is 1 on $K_{\rho}$.

Lemma 2. Assume that

$\left(\mathrm{I}_{\rho}^{1}\right)$ there exists $\rho>0$ such that for every $i=1,2$

$$
\frac{\left\|\gamma_{i 1}\right\|_{\infty} \beta_{i 1}\left[\gamma_{i 2}\right] \beta_{i 2}[1]}{\left(1-\beta_{i 1}\left[\gamma_{i 1}\right]\right)}+\left\|\gamma_{i 2}\right\|_{\infty} \beta_{i 2}[1]+f_{i}^{0, \rho}\left(\frac{1}{m_{i}}+\frac{\left\|\gamma_{i 1}\right\|_{\infty}}{\left(1-\beta_{i 1}\left[\gamma_{i 1}\right]\right)} \int_{0}^{1} \mathscr{K}_{i 1}(s) g_{i}(s) d s\right)<1
$$

where

$$
f_{i}^{0, \rho}=\sup \left\{\frac{f_{i}(t, u, v)}{\rho}:(t, u, v) \in[0,1] \times[0, \rho] \times[0, \rho]\right\} \quad \text { and } \quad \frac{1}{m_{i}}=\sup _{t \in[0,1]} \int_{0}^{1} k_{i}(t, s) g_{i}(s) d s .
$$

Then the fixed point index, $i_{K}\left(T, K_{\rho}\right)$, is equal to 1 .

Proof. We show that $\mu(u, v) \neq T(u, v)$ for every $(u, v) \in \partial K_{\rho}$ and for every $\mu \geqslant 1$; this ensures that the index is 1 on $K_{\rho}$. In fact, if this does not happen, there exist $\mu \geqslant 1$ and $(u, v) \in \partial K_{\rho}$ such that $\mu(u, v)=T(u, v)$. Assume, without loss of generality, that $\|u\|_{\infty}=\rho$ and $\|v\|_{\infty} \leqslant \rho$. Then

$$
\mu u(t)=\gamma_{11}(t) \beta_{11}[u]+\gamma_{12}(t) \beta_{12}[v]+F_{1}(u, v)(t)
$$

and therefore, since $v(t) \leqslant \rho$, for all $t \in[0,1]$,

$$
\mu u(t) \leqslant \gamma_{11}(t) \beta_{11}[u]+\gamma_{12}(t) \beta_{12}[\rho]+F_{1}(u, v)(t)=\gamma_{11}(t) \beta_{11}[u]+\rho \gamma_{12}(t) \beta_{12}[1]+F_{1}(u, v)(t)
$$

Applying $\beta_{11}$ to both sides of (10) gives

$$
\mu \beta_{11}[u] \leqslant \beta_{11}\left[\gamma_{11}\right] \beta_{11}[u]+\rho \beta_{11}\left[\gamma_{12}\right] \beta_{12}[1]+\beta_{11}\left[F_{1}(u, v)\right] .
$$

Thus we have

$$
\left(\mu-\beta_{11}\left[\gamma_{11}\right]\right) \beta_{11}[u] \leqslant \rho \beta_{11}\left[\gamma_{12}\right] \beta_{12}[1]+\beta_{11}\left[F_{1}(u, v)\right],
$$


that is

$$
\beta_{11}[u] \leqslant \rho \frac{\beta_{11}\left[\gamma_{12}\right] \beta_{12}[1]}{\left(\mu-\beta_{11}\left[\gamma_{11}\right]\right)}+\frac{\beta_{11}\left[F_{1}(u, v)\right]}{\left(\mu-\beta_{11}\left[\gamma_{11}\right]\right)}
$$

Substituting into (10) gives

$$
\begin{aligned}
\mu u(t) & \leqslant \gamma_{11}(t)\left(\rho \frac{\beta_{11}\left[\gamma_{12}\right] \beta_{12}[1]}{\left(\mu-\beta_{11}\left[\gamma_{11}\right]\right)}+\frac{\beta_{11}\left[F_{1}(u, v)\right]}{\left(\mu-\beta_{11}\left[\gamma_{11}\right]\right)}\right)+\rho \gamma_{12}(t) \beta_{12}[1]+F_{1}(u, v)(t) \\
& =\rho \frac{\gamma_{11}(t) \beta_{11}\left[\gamma_{12}\right] \beta_{12}[1]}{\left(\mu-\beta_{11}\left[\gamma_{11}\right]\right)}+\frac{\gamma_{11}(t)}{\left(\mu-\beta_{11}\left[\gamma_{11}\right]\right)} \int_{0}^{1} \mathscr{K}_{11}(s) g_{1}(s) f_{1}(s, u(s), v(s)) d s+\rho \gamma_{12}(t) \beta_{12}[1]+F_{1}(u, v)(t)
\end{aligned}
$$

Since $\mu \geqslant 1$, one has $\frac{1}{\mu-\beta_{11}\left[\gamma_{11}\right]} \leqslant \frac{1}{1-\beta_{11}\left[\gamma_{11}\right]}$ and therefore

$$
\mu u(t) \leqslant \rho \frac{\gamma_{11}(t) \beta_{11}\left[\gamma_{12}\right] \beta_{12}[1]}{\left(1-\beta_{11}\left[\gamma_{11}\right]\right)}+\frac{\gamma_{11}(t)}{\left(1-\beta_{11}\left[\gamma_{11}\right]\right)} \int_{0}^{1} \mathscr{K}_{11}(s) g_{1}(s) f_{1}(s, u(s), v(s)) d s+\rho \gamma_{12}(t) \beta_{12}[1]+F_{1}(u, v)(t)
$$

Taking the supremum of $t$ on $[0,1]$ gives

$$
\mu \rho \leqslant \rho \frac{\left\|\gamma_{11}\right\|_{\infty} \beta_{11}\left[\gamma_{12}\right] \beta_{12}[1]}{\left(1-\beta_{11}\left[\gamma_{11}\right]\right)}+\rho f_{1}^{0, \rho} \frac{\left\|\gamma_{11}\right\|_{\infty}}{\left(1-\beta_{11}\left[\gamma_{11}\right]\right)} \int_{0}^{1} \mathscr{K}_{11}(s) g_{1}(s) d s+\rho\left\|\gamma_{12}\right\|_{\infty} \beta_{12}[1]+\rho f_{1}^{0, \rho} \frac{1}{m_{1}}
$$

Using the hypothesis (9) we obtain $\mu \rho<\rho$. This contradicts the fact that $\mu \geqslant 1$ and proves the result.

We give a first Lemma that shows that the index is 0 on a set $V_{\rho}$, here we assume that the nonlinearities $f_{1}, f_{2}$ have the same growth.

\section{Lemma 3. Assume that}

$\left(\mathrm{I}_{\rho}^{0}\right)$ there exist $\rho>0$ such that for every $i=1,2$

$$
f_{i,(\rho, \rho / c)}\left(\frac{c_{i 1}\left\|\gamma_{i 1}\right\|_{\infty}}{\left(1-\beta_{i 1}\left[\gamma_{i 1}\right]\right)} \int_{a_{i}}^{b_{i}} \mathscr{K}_{i 1}(s) g_{i}(s) d s+\frac{1}{M_{i}}\right)>1,
$$

where

$$
\begin{aligned}
& f_{1,(\rho, \rho / c)}=\inf \left\{\frac{f_{1}(t, u, v)}{\rho}:(t, u, v) \in\left[a_{1}, b_{1}\right] \times[\rho, \rho / c] \times[0, \rho / c]\right\} \\
& f_{2,(\rho, \rho / c)}=\inf \left\{\frac{f_{2}(t, u, v)}{\rho}:(t, u, v) \in\left[a_{2}, b_{2}\right] \times[0, \rho / c] \times[\rho, \rho / c]\right\} \\
& \text { and } \frac{1}{M_{i}}=\inf _{t \in\left[a_{i}, b_{i}\right]} \int_{a_{i}}^{b_{i}} k_{i}(t, s) g_{i}(s) d s .
\end{aligned}
$$

Then $i_{K}\left(T, V_{\rho}\right)=0$.

Proof. Let $e(t) \equiv 1$ for $t \in[0,1]$. Then $(e, e) \in K$. We prove that $(u, v) \neq T(u, v)+\mu(e, e)$ for $(u, v) \in \partial V_{\rho}$ and $\mu \geqslant 0$.

In fact, if this does not happen, there exist $(u, v) \in \partial V_{\rho}$ and $\mu \geqslant 0$ such that $(u, v)=T(u, v)+\mu(e, e)$. Without loss of generality, we can assume that for all $t \in\left[a_{1}, b_{1}\right]$ we have

$$
\rho \leqslant u(t) \leqslant \rho / c, \quad \min u(t)=\rho \text { and } 0 \leqslant v(t) \leqslant \rho / c .
$$

Then, for $t \in\left[a_{1}, b_{1}\right]$, we obtain

$$
u(t)=\gamma_{11}(t) \beta_{11}[u]+\gamma_{12}(t) \beta_{12}[v]+\int_{0}^{1} k_{1}(t, s) g_{1}(s) f_{1}(s, u(s), v(s)) d s+\mu e(t)
$$

and therefore

$$
u(t) \geqslant \gamma_{11}(t) \beta_{11}[u]+F_{1}(u, v)(t)+\mu e(t) .
$$

Applying $\beta_{11}$ to both sides of (12) gives

$$
\beta_{11}[u] \geqslant \beta_{11}\left[\gamma_{11}\right] \beta_{11}[u]+\beta_{11}\left[F_{1}(u, v)\right]+\mu \beta_{11}[e] .
$$

This can be written in the form

$$
\left(1-\beta_{11}\left[\gamma_{11}\right]\right) \beta_{11}[u] \geqslant \beta_{11}\left[F_{1}(u, v)\right]+\mu \beta_{11}[e],
$$


that is

$$
\beta_{11}[u] \geqslant \frac{\beta_{11}\left[F_{1}(u, v)\right]}{\left(1-\beta_{11}\left[\gamma_{11}\right]\right)}+\frac{\mu \beta_{11}[e]}{\left(1-\beta_{11}\left[\gamma_{11}\right]\right)} .
$$

Thus, (12) becomes

$$
\begin{aligned}
u(t) & \geqslant \frac{\gamma_{11}(t) \beta_{11}\left[F_{1}(u, v)\right]}{\left(1-\beta_{11}\left[\gamma_{11}\right]\right)}+\frac{\mu \gamma_{11}(t) \beta_{11}[e]}{\left(1-\beta_{11}\left[\gamma_{11}\right]\right)}+F_{1}(u, v)(t)+\mu e(t) \\
& =\frac{\gamma_{11}(t)}{\left(1-\beta_{11}\left[\gamma_{11}\right]\right)} \times \int_{0}^{1} \mathscr{K}_{11}(s) g_{1}(s) f_{1}(s, u(s), v(s)) d s+\frac{\mu \gamma_{11}(t) \beta_{11}[e]}{\left(1-\beta_{11}\left[\gamma_{11}\right]\right)}+\int_{0}^{1} k_{1}(t, s) g_{1}(s) f_{1}(s, u(s), v(s)) d s+\mu .
\end{aligned}
$$

Then we have, for $t \in\left[a_{1}, b_{1}\right]$,

$$
\begin{aligned}
u(t) & \geqslant \frac{c_{11}\left\|\gamma_{11}\right\|_{\infty}}{\left(1-\beta_{11}\left[\gamma_{11}\right]\right)} \times \int_{a_{1}}^{b_{1}} \mathscr{K}_{11}(s) g_{1}(s) f_{1}(s, u(s), v(s)) d s+\frac{\mu c_{11}\left\|\gamma_{11}\right\|_{\infty} \beta_{11}[e]}{\left(1-\beta_{11}\left[\gamma_{11}\right]\right)}+\int_{a_{1}}^{b_{1}} k_{1}(t, s) g_{1}(s) f_{1}(s, u(s), v(s)) d s+\mu \\
& \geqslant \frac{c_{11}\left\|\gamma_{11}\right\|_{\infty}}{\left(1-\beta_{11}\left[\gamma_{11}\right]\right)} \times \int_{a_{1}}^{b_{1}} \mathscr{K}_{11}(s) g_{1}(s) f_{1}(s, u(s), v(s)) d s+\int_{a_{1}}^{b_{1}} k_{1}(t, s) g_{1}(s) f_{1}(s, u(s), v(s)) d s+\mu
\end{aligned}
$$

Taking the minimum over $\left[a_{1}, b_{1}\right]$ gives

$$
\begin{aligned}
\min _{t \in\left[a_{1}, b_{1}\right]} u(t) & \geqslant \rho f_{1,(\rho, \rho / c)} \frac{c_{11}\left\|\gamma_{11}\right\|_{\infty}}{\left(1-\beta_{11}\left[\gamma_{11}\right]\right)} \times \int_{a_{1}}^{b_{1}} \mathscr{K}_{11}(s) g_{1}(s) d s+\rho f_{1,(\rho, \rho / c)} \frac{1}{M_{1}}+\mu \\
& =\rho\left(f_{1,(\rho, \rho / c)} \frac{c_{11}\left\|\gamma_{11}\right\|_{\infty}}{\left(1-\beta_{11}\left[\gamma_{11}\right]\right)} \times \int_{a_{1}}^{b_{1}} \mathscr{K}_{11}(s) g_{1}(s) d s+f_{1,(\rho, \rho / c)} \frac{1}{M_{1}}\right)+\mu .
\end{aligned}
$$

Using the hypothesis (11) we obtain $\rho=\min _{t \in\left[a_{1}, b_{1}\right]} u(t)>\rho+\mu$, a contradiction.

The following Lemma also shows that the index is 0 on $V_{\rho}$; the idea here is similar to the one in Lemma 4 of [16]: this time we have to control the growth of just one nonlinearity $f_{i}$, at the cost of having to deal with a larger domain. For other results on the existence of solutions with different growth on the nonlinearities see the works [28,29] and the paper by Yang [35].

Lemma 4. Assume that

$\left(\mathrm{I}_{\rho}^{0}\right)^{\star}$ there exist $\rho>0$ such that for some $i=1,2$

$$
f_{i,(0, \rho / c)}^{*}\left(\frac{c_{i 1}\left\|\gamma_{i 1}\right\|_{\infty}}{\left(1-\beta_{i 1}\left[\gamma_{i 1}\right]\right)} \int_{a_{i}}^{b_{i}} \mathscr{K}_{i 1}(s) g_{i}(s) d s+\frac{1}{M_{i}}\right)>1,
$$

where

$$
f_{i,(0, \rho / c)}^{*}=\inf \left\{\frac{f_{i}(t, u, v)}{\rho}:(t, u, v) \in\left[a_{i}, b_{i}\right] \times[0, \rho / c] \times[0, \rho / c]\right\} .
$$

Then $i_{K}\left(T, V_{\rho}\right)=0$.

Proof. Suppose that the condition (13) holds for $i=1$. Let $e(t) \equiv 1$ for $t \in[0,1]$. Then $(e, e) \in K$. We prove that

$(u, v) \neq T(u, v)+\mu(e, e)$ for $(u, v) \in \partial V_{\rho}$ and $\mu \geqslant 0$.

In fact, if this does not happen, there exist $(u, v) \in \partial V_{\rho}$ and $\mu \geqslant 0$ such that $(u, v)=T(u, v)+\mu(e, e)$. So, for all $t \in\left[a_{1}, b_{1}\right], \min u(t) \leqslant \rho$ and for $t \in\left[a_{2}, b_{2}\right], \min v(t) \leqslant \rho$. We have, for $t \in[0,1]$,

$$
u(t)=\gamma_{11}(t) \beta_{11}[u]+\gamma_{12}(t) \beta_{12}[v]+\int_{0}^{1} k_{1}(t, s) g_{1}(s) f_{1}(s, u(s), v(s)) d s+\mu e(t)
$$

and, as in the proof of Lemma 3,

$$
u(t) \geqslant \frac{\gamma_{11}(t)}{\left(1-\beta_{11}\left[\gamma_{11}\right]\right)} \times \int_{0}^{1} \mathscr{K}_{11}(s) g_{1}(s) f_{1}(s, u(s), v(s)) d s+\frac{\mu \gamma_{11}(t) \beta_{11}[e]}{\left(1-\beta_{11}\left[\gamma_{11}\right]\right)}+\int_{0}^{1} k_{1}(t, s) g_{1}(s) f_{1}(s, u(s), v(s)) d s+\mu
$$

Then we have

$$
\begin{aligned}
\min _{t \in\left[a_{1}, b_{1}\right]} u(t) & \geqslant \rho f_{1,(0, \rho / c)}^{*} \frac{c_{11}\left\|\gamma_{11}\right\|_{\infty}}{\left(1-\beta_{11}\left[\gamma_{11}\right]\right)} \times \int_{a_{1}}^{b_{1}} \mathscr{K}_{11}(s) g_{1}(s) d s+\rho f_{1,(0, \rho / c)}^{*} \frac{1}{M_{1}}+\mu \\
& =\rho f_{1,(0, \rho / c)}^{*} \frac{c_{11}\left\|\gamma_{11}\right\|_{\infty}}{\left(1-\beta_{11}\left[\gamma_{11}\right]\right)} \times \int_{a_{1}}^{b_{1}} \mathscr{K}_{11}(s) g_{1}(s) d s+\rho f_{1,(0, \rho / c)}^{*} \frac{1}{M_{1}}+\mu
\end{aligned}
$$


Using the hypothesis (13) we obtain $\min _{t \in\left[a_{1}, b_{1}\right]} u(t)>\rho+\mu \geqslant \rho$, a contradiction.

The above Lemmas can be combined to prove the following Theorem, here we deal with the existence of at least one, two or three solutions. We stress that, by expanding the lists in conditions $\left(S_{5}\right),\left(S_{6}\right)$ below, it is possible to state results for four or more positive solutions, see for example the paper by Lan [21] for the type of results that might be stated. We omit the proof which follows from the properties of fixed point index.

Theorem 5. The system (7) has at least one positive solution in $K$ if either of the following conditions hold.

$\left(S_{1}\right)$ There exist $\rho_{1}, \rho_{2} \in(0, \infty)$ with $\rho_{1} / c<\rho_{2}$ such that $\left(\mathrm{I}_{\rho_{1}}^{0}\right)\left[\right.$ or $\left.\left(\mathrm{I}_{\rho_{1}}^{0}\right)^{\star}\right],\left(\mathrm{I}_{\rho_{2}}^{1}\right)$ hold.

$\left(S_{2}\right)$ There exist $\rho_{1}, \rho_{2} \in(0, \infty)$ with $\rho_{1}<\rho_{2}$ such that $\left(\mathrm{I}_{\rho_{1}}^{1}\right)$, $\left(\mathrm{I}_{\rho_{2}}^{0}\right)$ hold.

The system (7) has at least two positive solutions in $K$ if one of the following conditions hold:

$\left(S_{3}\right)$ There exist $\rho_{1}, \rho_{2}, \rho_{3} \in(0, \infty)$ with $\rho_{1} / c<\rho_{2}<\rho_{3}$ such that $\left(\mathrm{I}_{\rho_{1}}^{0}\right)\left[\right.$ or $\left.\left(\mathrm{I}_{\rho_{1}}^{0}\right)^{\star}\right],\left(\mathrm{I}_{\rho_{2}}^{1}\right)$ and $\left(\mathrm{I}_{\rho_{3}}^{0}\right)$ hold.

$\left(S_{4}\right)$ There exist $\rho_{1}, \rho_{2}, \rho_{3} \in(0, \infty)$ with $\rho_{1}<\rho_{2}$ and $\rho_{2} / c<\rho_{3}$ such that $\left(\mathrm{I}_{\rho_{1}}^{1}\right),\left(\mathrm{I}_{\rho_{2}}^{0}\right)$ and $\left(\mathrm{I}_{\rho_{3}}^{1}\right)$ hold.

The system (7) has at least three positive solutions in $K$ if one of the following conditions hold:

$\left(S_{5}\right)$ There exist $\rho_{1}, \rho_{2}, \rho_{3}, \rho_{4} \in(0, \infty)$ with $\rho_{1} / c<\rho_{2}<\rho_{3}$ and $\rho_{3} / c<\rho_{4}$ such that $\left(\mathrm{I}_{\rho_{1}}^{0}\right)\left[\right.$ or $\left.\left(\mathrm{I}_{\rho_{1}}^{0}\right)^{\star}\right],\left(\mathrm{I}_{\rho_{2}}^{1}\right)$, ( $\left.\mathrm{I}_{\rho_{3}}^{0}\right)$ and $\left(\mathrm{I}_{\rho_{4}}^{1}\right)$ hold.

$\left(S_{6}\right)$ There exist $\rho_{1}, \rho_{2}, \rho_{3}, \rho_{4} \in(0, \infty)$ with $\rho_{1}<\rho_{2}$ and $\rho_{2} / c<\rho_{3}<\rho_{4}$ such that $\left(\mathrm{I}_{\rho_{1}}^{1}\right),\left(\mathrm{I}_{\rho_{2}}^{0}\right),\left(\mathrm{I}_{\rho_{3}}^{1}\right)$ and $\left(\mathrm{I}_{\rho_{4}}^{0}\right)$ hold.

Remark 1. Note that, if the nonlinearities $f_{1}$ and $f_{2}$ have some extra positivity properties, a solution $(u, v)$ achieved by means of the above Theorem has further positivity properties. For example, if the condition $\left(S_{1}\right)$ holds and moreover we assume that $f_{1}(t, 0, v)>0$ in $\left[a_{1}, b_{1}\right] \times\{0\} \times\left[0, \rho_{2}\right]$ and $f_{2}(t, u, 0)>0$ in $\left[a_{2}, b_{2}\right] \times\left[0, \rho_{2}\right] \times\{0\}$, the solution $(u, v)$ of the system $(7)$ is such that $\|u\|_{\infty}$ and $\|v\|_{\infty}$ are strictly positive. The Remark 2 of [16] should read accordingly.

\section{An application to coupled systems of BVPs}

We study the existence of positive solutions for the system of second order differential equations

$$
\begin{aligned}
& u^{\prime \prime}(t)+g_{1}(t) f_{1}(t, u(t), v(t))=0, \quad t \in(0,1), \\
& v^{(4)}(t)=g_{2}(t) f_{2}(t, u(t), v(t)), \quad t \in(0,1),
\end{aligned}
$$

with the nonlocal boundary conditions

$$
\begin{aligned}
& u(0)=\beta_{11}[u], \quad u(1)=\beta_{12}[v], \\
& v(0)=\beta_{21}[v], \quad v(1)=0, \quad v^{\prime \prime}(0)=0, \quad v^{\prime \prime}(1)+\beta_{22}[u]=0 .
\end{aligned}
$$

We rewrite this differential system in the integral form

$$
\begin{aligned}
& u(t)=(1-t) \beta_{11}[u]+t \beta_{12}[u]+\int_{0}^{1} k_{1}(t, s) g_{1}(s) f_{1}(s, u(s), v(s)) d s, \\
& v(t)=(1-t) \beta_{21}[v]+\frac{1}{6} t\left(1-t^{2}\right) \beta_{22}[u]+\int_{0}^{1} k_{2}(t, s) g_{2}(s) f_{2}(s, u(s), v(s)) d s,
\end{aligned}
$$

where

$$
k_{1}(t, s)=\left\{\begin{array}{ll}
s(1-t), & s \leqslant t, \\
t(1-s), & s>t,
\end{array} \quad \text { and } \quad k_{2}(t, s)= \begin{cases}\frac{1}{6} s(1-t)\left(2 t-s^{2}-t^{2}\right), & s \leqslant t \\
\frac{1}{6} t(1-s)\left(2 s-t^{2}-s^{2}\right), & s>t\end{cases}\right.
$$

are non-negative continuous functions on $[0,1] \times[0,1]$.

The intervals $\left[a_{1}, b_{1}\right]$ and $\left[a_{2}, b_{2}\right]$ may be chosen arbitrarily in $(0,1)$. It is easy to check that

$$
k_{1}(t, s) \leqslant s(1-s):=\Phi_{1}(s), \quad \min _{t \in\left[a_{1}, b_{1}\right]} k_{1}(t, s) \geqslant c_{1} s(1-s),
$$

where $c_{1}=\min \left\{1-b_{1}, a_{1}\right\}$. Furthermore, see the paper by Webb et al. [34], we have that

$$
k_{2}(t, s) \leqslant \Phi_{2}(s):= \begin{cases}\frac{\sqrt{3}}{27} s\left(1-s^{2}\right)^{\frac{3}{2}}, & \text { for } 0 \leqslant s \leqslant \frac{1}{2} \\ \frac{\sqrt{3}}{27}(1-s) s^{\frac{3}{2}}(2-s)^{\frac{3}{2}}, & \text { for } \frac{1}{2}<s \leqslant 1\end{cases}
$$

and

$$
k_{2}(t, s) \geqslant c_{2}(t) \Phi_{2}(s)
$$

where 


$$
c_{2}(t)= \begin{cases}\frac{3 \sqrt{3}}{2} t\left(1-t^{2}\right), & \text { for } t \in[0,1 / 2], \\ \frac{3 \sqrt{3}}{2} t(1-t)(2-t), & \text { for } t \in(1 / 2,1]\end{cases}
$$

so that

$$
c_{2}=\min _{t \in\left[a_{2}, b_{2}\right]} c_{2}(t)>0 .
$$

The existence of multiple solutions of the system (14)-(15) follows from Theorem 5.

In the next example we illustrate the constants that occur in our theory.

Example 1. Consider the system

$$
\begin{aligned}
& u \prime \prime+(1 / 8)\left(u^{3}+t^{3} v^{3}\right)+2=0, \quad t \in(0,1), \\
& v^{(4)}=\sqrt{t u}+13 v^{2}, \quad t \in(0,1), \\
& u(0)=(1 / 2) u(1 / 4), u(1)=(1 / 3) v(3 / 4), \\
& v(0)=(1 / 4) v(1 / 3), \quad v(1)=v^{\prime \prime}(0)=0, \quad v^{\prime \prime}(1)+(1 / 5) u(2 / 3)=0 .
\end{aligned}
$$

In this case the nonlocal conditions are given by the functionals $\beta_{i j}[w]=\delta_{i j} w\left(\eta_{i j}\right)$.

The choice $\left[a_{1}, b_{1}\right]=\left[a_{2}, b_{2}\right]=[1 / 4,3 / 4]$ gives

$$
\begin{aligned}
& c_{1}=1 / 4, \quad c_{2}=45 \sqrt{3} / 128, \quad c_{11}=c_{12}=c_{21}=1 / 4, \quad c_{22}=45 \sqrt{3} / 128, \\
& m_{1}=8, \quad M_{1}=16, \quad m_{2}=384 / 5, \quad M_{2}=768 / 5 .
\end{aligned}
$$

We have that $\beta_{11}\left[\gamma_{11}\right]=3 / 8$ and $\beta_{21}\left[\gamma_{21}\right]=1 / 6$.

Since $\mathscr{K}_{i 1}(s)=\delta_{i 1} k_{i}\left(\eta_{i 1}, s\right)$ we obtain

$$
\begin{aligned}
& \int_{0}^{1} \mathscr{K}_{11}(s) d s=3 / 64 \text { and } \int_{1 / 4}^{3 / 4} \mathscr{K}_{11}(s) d s=1 / 32 \\
& \int_{0}^{1} \mathscr{K}_{21}(s) d s=11 / 3888 \text { and } \int_{1 / 4}^{3 / 4} \mathscr{K}_{21}(s) d s=3985 / 1990656 .
\end{aligned}
$$
exact)

Then, for $\rho_{1}=1 / 8, \rho_{2}=1$ and $\rho_{3}=11$, we have (the constants that follow have been rounded to 2 decimal places unless

$$
\begin{aligned}
& \inf \left\{f_{1}(t, u, v):(t, u, v) \in[1 / 4,3 / 4] \times[0,1 / 2] \times[0,1 / 2]\right\}=f_{1}(1 / 4,0,0)>13.34 \rho_{1}, \\
& \sup \left\{f_{1}(t, u, v):(t, u, v) \in[0,1] \times[0,1] \times[0,1]\right\}=f_{1}(1,1,1)<3 \rho_{2}, \\
& \sup \left\{f_{2}(t, u, v):(t, u, v) \in[0,1] \times[0,1] \times[0,1]\right\}=f_{2}(1,1,1)<59.95 \rho_{2}, \\
& \inf \left\{f_{1}(t, u, v):(t, u, v) \in[1 / 4,3 / 4] \times[11,44] \times[0,44]\right\}=f_{1}(1 / 4,11,0)>13.34 \rho_{3}, \\
& \inf \left\{f_{2}(t, u, v):(t, u, v) \in[1 / 4,3 / 4] \times[0,44] \times[11,44]\right\}=f_{2}(1 / 4,0,11)>140.63 \rho_{3},
\end{aligned}
$$

that is the conditions $\left(\mathrm{I}_{\rho_{1}}^{0}\right)^{\star},\left(\mathrm{I}_{\rho_{2}}^{1}\right)$ and $\left(\mathrm{I}_{\rho_{3}}^{0}\right)$ are satisfied; therefore the system (16) has at least two positive solutions in $K$.

\section{References}

[1] Agarwal RP, O’Regan D, Wong PJY. Constant-sign solutions of a system of integral equations with integrable singularities. J Integr Eq Appl 2007; $19: 117-42$.

[2] Agarwal RP, O'Regan D, Wong PJY. Constant-sign solutions for singular systems of Fredholm integral equations. Math Methods Appl Sci 2010;33:1783-93.

[3] Agarwal RP, O'Regan D, Wong PJY. Constant-sign solutions for systems of singular integral equations of Hammerstein type. Math Comput Model 2009;50:999-1025.

[4] Ahmad B, Graef JR. Coupled systems of nonlinear fractional differential equations with nonlocal boundary conditions. Panamer Math J 2009;19:29-39.

[5] Ahmad B, Nieto JJ. Existence results for a coupled system of nonlinear fractional differential equations with three-point boundary conditions. Comput Math Appl 2009;58:1838-43.

[6] Amann H. Fixed point equations and nonlinear eigenvalue problems in ordered Banach spaces. SIAM Rev 1976;18:620-709.

[7] Amann H. Parabolic evolution equations with nonlinear boundary conditions, Part 1 (Berkeley, CA, 1983). p. 17-27. Proc. Sympos. Pure Math., vol. 45, Part 1. Providence, RI: Amer. Math. Soc.; 1986.

[8] Asif NA, Khan RA. Positive solutions to singular system with four-point coupled boundary conditions. J Math Anal Appl 2012;386:848-61.

[9] Cui Y, Sun J. On existence of positive solutions of coupled integral boundary value problems for a nonlinear singular superlinear differential system. Electron J Qual Theory Differ Eq No 2012;41:1-13.

[10] Franco D, Infante G, O’Regan D. Nontrivial solutions in abstract cones for Hammerstein integral systems. Dyn Contin Discrete Impuls Syst Ser A: Math Anal 14 (2007) 837-50.

[11] Goodrich CS. Nonlocal systems of BVPs with asymptotically superlinear boundary conditions. Comment Math Univ Carolin 2012;53:79-97.

[12] Goodrich CS. Nonlocal systems of BVPs with asymptotically sublinear boundary conditions. Appl Anal Discrete Math, in press. http://dx.doi.org/ 10.2298/AADM120329010G.

[13] Guo D, Lakshmikantham V. Nonlinear problems in abstract cones. Academic Press; 1988. 
[14] Henderson J, Ntouyas SK, Purnaras IK. Positive solutions for systems of second order four-point nonlinear boundary value problems. Commun Appl Anal 2008;12:29-40.

[15] Infante G, Pietramala P. Eigenvalues and non-negative solutions of a system with nonlocal BCs. Nonlinear Stud 2009;16:187-96.

[16] Infante G, Pietramala P. Existence and multiplicity of non-negative solutions for systems of perturbed Hammerstein integral equations. Nonlinear Anal 2009;71:1301-10.

[17] Infante G, Webb JRL. Nonlinear nonlocal boundary value problems and perturbed Hammerstein integral equations. Proc Edinb Math Soc 2006;49:637-56.

[18] Kang P, Wei Z. Three positive solutions of singular nonlocal boundary value problems for systems of nonlinear second-order ordinary differential equations. Nonlinear Anal 2009;70:444-51.

[19] Karakostas GL, Tsamatos PCh. Existence of multiple positive solutions for a nonlocal boundary value problem. Topol Methods Nonlinear Anal 2002;19:109-21.

[20] Lazer AC, McKenna PJ. Large-amplitude periodic oscillations in suspension bridges: some new connections with nonlinear analysis. SIAM Rev 1990;32:537-78.

[21] Lan KQ. Multiple positive solutions of Hammerstein integral equations with singularities. Diff Eq Dynam Syst 2000;8:175-95.

[22] Lan KQ. Multiple positive solutions of semilinear differential equations with singularities. J Lond Math Soc 2001;63:690-704.

[23] Lan KQ, Lin W. Multiple positive solutions of systems of Hammerstein integral equations with applications to fractional differential equations. J Lond Math Soc 2011;83:449-69.

[24] Leung A. A semilinear reaction-diffusion prey-predator system with nonlinear coupled boundary conditions: equilibrium and stability. Indiana Univ Math J 1982;31:223-41.

[25] Lü H, Yu H, Liu Y. Positive solutions for singular boundary value problems of a coupled system of differential equations. J Math Anal Appl 2005;302:14-29.

[26] Matas A. Mathematical models of suspension bridges. PhD thesis, University of West Bohemia in Pilsen, Pilsen; 2004.

[27] Mehmeti FA, Nicaise S. Nonlinear interaction problems. Nonlinear Anal 1993;20:27-61.

[28] Precup R. Componentwise compression-expansion conditions for systems of nonlinear operator equations and applications. Math Model Eng Biol Med. p. 284-93. AIP Conf. Proc., vol. 1124. Melville, NY: Amer. Inst. Phys.; 2009.

[29] Precup R. Existence, localization and multiplicity results for positive radial solutions of semilinear elliptic systems. J Math Anal Appl 2009;352:48-56.

[30] Sun Y. Necessary and sufficient condition for the existence of positive solutions of a coupled system for elastic beam equations. J Math Anal Appl 2009;357:77-88

[31] Webb JRL. Solutions of nonlinear equations in cones and positive linear operators. J Lond Math Soc 2010;82:420-36.

[32] Webb JRL, Infante G. Positive solutions of nonlocal boundary value problems: a unified approach. J Lond Math Soc 2006;74:673-93.

[33] Webb JRL, Infante G. Nonlocal boundary value problems of arbitrary order. J Lond Math Soc 2009;79:238-58.

[34] Webb JRL, Infante G, Franco D. Positive solutions of nonlinear fourth-order boundary-value problems with local and non-local boundary conditions. Proc R Soc Edin Sect A 2008;138:427-46.

[35] Yang Z. Positive solutions to a system of second-order nonlocal boundary value problems. Nonlinear Anal 2005;62:1251-65.

[36] Yang Z, Kong L. Positive solutions of a system of second order boundary value problems involving first order derivatives via $R_{+}^{n}$-monotone matrices. Nonlinear Anal 2012;75:2037-46.

[37] Yang Z, Zhang Z. Positive solutions for a system of nonlinear singular Hammerstein integral equations via nonnegative matrices and applications. Positivity, in press. http://dx.doi.org/10.1007/s11117-011-0146-4.

[38] Yuan C, Jiang D, O'Regan D, Agarwal RP. Multiple positive solutions to systems of nonlinear semipositone fractional differential equations with coupled boundary conditions. Electron J Qual Theory Differ Eq No 2012;13:1-13. 